\title{
Removal Mechanism and Defect Characterization for Glass- Side Laser Scribing of CdTe/CdS Multilayer in Solar Cells
}

\author{
Hongliang Wang, Y. Lawrence Yao \\ Department of Mechanical Engineering \\ Columbia University \\ New York, NY, USA \\ Hongqiang Chen \\ Laser \& Metrology System Lab \\ GE Global Research \\ Niskayuna, NY, USA
}

\begin{abstract}
Laser scribing is an important manufacturing process used to reduce photocurrent and resistance losses and increase solar cell efficiency through the formation of serial interconnections in large-area solar cells. High-quality scribing is crucial since the main impediment to large-scale adoption of solar power is its high production cost (price-per-watt) compared to competing energy sources such as wind and fossil fuels. In recent years, the use of glass-side laser scribing processes has led to increased scribe quality and solar cell efficiencies, however, defects introduced during the process such as thermal effect, micro cracks, film delamination, and removal uncleanliness keep the modules from reaching their theoretical efficiencies. Moreover, limited numerical work has been performed in predicting thin film laser removal processes. In this study, a nanosecond (ns) laser with a wavelength at $532 \mathrm{~nm}$ is employed for pattern 2 (P2) scribing on CdTe (Cadmium telluride) based thin-film solar cells. The film removal mechanism and defects caused by laser-induced micro-explosion process are studied. The relationship between those defects, removal geometry, laser fluences and scribing speeds are also investigated. Thermal and mechanical numerical models are developed to analyze the laser-induced spatio-temporal temperature and pressure responsible for film removal. The simulation can well-predict the film removal geometries, generation of micro cracks, film delamination and remaining materials. The characterization of removal qualities will enable the process optimization and design required to enhance solar module efficiency.
\end{abstract}

\section{KEYWORDS}

Glass-side laser scribing, micro-explosion, multilayer thin films, CdTe, solar cell

\section{INTRODUCTION}

Thin-film solar cell technology promises to achieve a significant cost reduction in materials, by adopting large area deposition capability, and the use of cheap and flexible substrates. Typical thin film solar cells used in terrestrial PV (photovoltaic) applications consist of back contact, absorber and front contact films. CdTe is the dominant absorber material in recent years because of its attractive price and stable performance at high temperatures [1-2]. The efficiency of thin-film solar panels, however, is hampered by resistive losses in the module proportional to the square of the photocurrent. In practice, photocurrent is decreased by scribing the solar module into a large number (between 100 and 200) mini-modules and connecting them in series to create high-voltage, low-current devices [3]. Since each layer in the solar module must be scribed after deposition, scribing is performed in 3 steps - Patterns 1, 2 and 3 (P1, P2 and $\mathrm{P} 3$ ) processes, which are also used in the commercial production of a-Si:H (hydrogenated amorphous silicon) and $\mathrm{CI}(\mathrm{G}) \mathrm{S}$ (copper indium gallium selenide) based thin film solar cell fabrications [4-6]. Compared to mechanical scribing, key advantage of laser scribing is able to enable much smaller line width $(50 \mu \mathrm{m}$ Vs. $500 \mu \mathrm{m})$, so the "dead zone" can be much smaller with higher efficiency. Also, it is currently only industrial standard process for high speed mass production (scribing speed around $1 \mathrm{~m} / \mathrm{s}$ Vs $0.05 \sim 0.1 \mathrm{~m} / \mathrm{s}$ ). However, laser scribing has been shown to leave a heat-affected zone around the scribe, which causes undesirably poor isolation between cells and low shunt resistance. Laser scribing has also been shown to leave high protruded ridges along the edge of the scribe line, contributing to electrical shorts [4]. While scribing reduces resistive losses by decreasing photocurrent, it also 
forms dead zones between P1 and P3 slots, which contribute to reductions in module efficiency [7].

In order to decrease the thermal effect of laser irradiation during processing, the use of ultrashort pulsed lasers, such as picosecond and femtosecond lasers, are being investigation for scribing processes [8-9]. These lasers are complex and expensive, and regardless of pulse duration, material melting cannot be totally eliminated [5]. Glass side laser processing [10-11] has been shown to be more efficient than film side processing with reduced thermal effect. Film side laser scribing is governed by heating, melting and vaporizing of selective films, while glass side laser scribing is a thermal-mechanical process which involves stress induced material failure and removal rather than vaporization. The mechanical fracture and removal of film material during glass side scribing is commonly referred to as lift off or micro-explosion processing [7]. During micro-explosion processing, the laser irradiates through the transparent substrate and is fully absorbed in a very thin layer of film at the interface. High pressure plasma is generated and expanded in the film. The plasma punches through the solid film above and the material is removed mechanically [12]. Micro-explosion processing is pronounced when the laser material penetration depth is much shallower than the film thickness, such as $\mathrm{P} 2 / \mathrm{P} 3$ processes of $\mathrm{CdTe}$ and a-Si:H solar cells. Otherwise, films are removed through thermal ablation, such as P1 process for front contact films made by transparent conducting oxide (TCO) materials [13].

While glass-side laser scribing has led to improved scribe quality over competing methods, defects such as thermal effect, film delamination, scribe uncleanliness and micro cracks that lead to decreased module efficiency are still introduced [14-15]. Although extensive work is carried out for optimizing the scribing qualities [4-13], the physical phenomena responsible for film removal during laser scribing and their effect on scribe quality are not well known. In addition, development of simulation capabilities will enable the fundamental understanding of the physical mechanisms and optimize the scribing processes instead of relying on trial-and-error experiments. To date, only rudimentary modeling efforts have been made, offering no predictive or optimization capabilities. Wang et al. [13] developed two-dimensional thermal model to estimate the temperature distribution of CdTe-based thin film solar cells by ns laser pulse irradiated from the glass side, and simulate the film removal during micro-explosion process. However, the model does not consider the dynamic response of brittle materials during this high-velocity shock wave impact, and it also offers no experimental validations.

Based on the current thin-film solar cell technology, a $1 \%$ increase in efficiency from improved scribe quality equates to roughly a $10 \%$ reduction cost. Therefore, understanding the film removal mechanisms and characterizing the potential defects during laser scribing processes are important for the cost reduction of thin film solar cells. In the previous work [13], P1 scribing of TCO layer has been studied. P2 and P3 are similar processes, since both are under micro-explosion mechanism with the plasma occurs at the interface between absorber and TCO layers. In this paper, P2 scribing of CdTe-based solar cells is carried out using a 532nm-wavelength 9ns-pulse-duration laser irradiated from glass side at different fluences and scribing speeds, and the corresponding defects are characterized. A two-dimensional numerical model, implemented with dynamic response of brittle material, is developed to simulate film removal via a micro-explosion process. The scribe geometry is studied by scanning electron microscopy (SEM) and optical profilometry, and removal cleanliness is estimated by energy-dispersive $\mathrm{x}$-ray spectroscopy (EDX). Film microstructures and laserinduced defects are characterized by transmission electron microscopy (TEM).

\section{BACKGROUND}

\section{Laser induced defects in solar cells}

Laser processing has been incorporated into many successful modifications of the properties of semiconductor materials in solar cells applications. For example, Tull et al. [16] and Wang et al. [17-18] have reported laser surface texturing induced optical property change of crystalline silicon and amorphous silicon. Compaan et al. [4] has investigated laser scribing on different contact and absorber layers in order to change their electrical property. However, the quality of the laser treatment is often limited by the generation of defects which have adverse effects on solar cell performance through increased junction shunting or recombination centers. Due to the unique mechanical fracture caused by the micro-explosion process, defects as atomic structural change, micro cracks/voids and film delamination are induced resulting in poor photocurrent and inactive cells. Kontgers et al. [15] have investigated the risk of power loss, up to $10 \%$, in photovoltaic modules due to micro cracks induced inactive cells, and pointed out that the micro cracks can be continually opened up subjected to the mechanical and thermal stress. Acciani et al. [19] have examined the abnormal heating in the restricted area of solar cells due to the presence of defects, such as voids, causes hot-spots, and the increased temperature will introduce dead cells in the solar panel.

Defects generation can also be introduced by laserinduced thermal effect. Golovan et al. [20] investigate the defect formation in $\mathrm{CdTe}$ under laser-induced melting process and showed a decrease of photoreflectance due to the formation of electrically and recombination active defects. In addition, when the laser photon energy is higher than the band gap of semiconductors, defect formation of recombination centers can be introduced even at low laser fluences (less than the melting threshold). Emel'yanov et al. [21] show that the fraction of the energy of the photo- 
excited electron-hole pairs is transferred in the process of electron-phonon relaxation and heat the lattice. The strong rise in temperature $T$ (up to melting point) leads to the generation of defects by thermo-fluctuation, which are frozen in after the end of the pulse due to the very rapid cooling process. The density of the thermo-fluctuation defects reaches its stationary value

$$
N_{d}=\text { const } \cdot \exp \left[-\frac{E_{A}}{K\left(T_{0}+\Delta T\right)}\right]
$$

where $T_{0}$ is the initial temperature, $\Delta T$ is the temperature rise due to laser heating, $E_{A}$ is the defect formation energy, $K$ is Boltzmann constant. This equation describes the generation of vacancies (Schottky defects), the annihilation and the formation of defect complexes and other defects [21]. The lattice deformation caused by the heating reduces the value of the defect activation energy in Eq. (1). The defect formation energy can also be reduced due to the localization of the electronic energy in some point of the crystal, which will decrease the potential barrier of the defect transition to a new equilibrium position. Thus, the localization of the electronic excitation on some initial defect can introduce new centers due to the process of defect multiplication.

\section{Dynamic response of brittle materials}

Due to the short duration of the laser pulse, film removal always occurs in nanosecond scales. This highvelocity pressure impact on CdTe will cause a high strain rate during laser scribing. Therefore, an assessment of the inelastic behavior including strain-rate phenomena, material degradation and pressure hardening needs to be considered. Micromechanical-based approach typically starts with the behavior of a single defect (crack or void) and the continuum level model is obtained by applying statistical averaging to an ensemble of defects [22]. Johnson and Holmquist (JH) [23-24] reported extensive work on brittle material response to high velocity impacts. The JH models use experimental data to determine constants that describe inelastic material behaviors in the simulation, and they are more convenient to be implemented in finite-element models than the theoretical statistical calculation.

There are two variations of the $\mathrm{JH}$ models. Compared to the first version (JH-1), the second version (JH-2) incorporates a damage evolution rule by consideration of progressive damage with increasing deformation, which is more accurate for simulating the high-velocity impact performance of ceramics [23]. In JH-2 model, the strength of the material is expressed in terms of the normalized von Mises equivalent stress as

$$
\sigma^{*}=\sigma_{i}^{*}-D\left(\sigma_{i}^{*}-\sigma_{f}^{*}\right)
$$

where $\sigma_{i}^{*}$ is the normalized intact equivalent stress, $\sigma_{f}^{*}$ is the normalized fractured equivalent stress, and $D$ is the damage variable. The normalized equivalent stresses $\left(\sigma^{*}\right.$, $\sigma_{i}^{*}, \sigma_{f}^{*}$ ) have the general form $\sigma^{*}=\sigma / \sigma_{\text {HEL }}$, where $\sigma$ is the actual von Mises equivalent stress and $\sigma_{H E L}$ is the equivalent stress at the Hugoniot elastic limit (HEL). The model assumes that the normalized intact and fractured stress can be expressed as function of the pressure and strain rate as

$$
\begin{aligned}
& \sigma_{\mathrm{i}}^{*}=A\left(p^{*}+T^{*}\right)^{N}\left[1+C \ln \dot{\varepsilon}^{*}\right] \leq \sigma_{\mathrm{i}}^{\max } \\
& \sigma_{f}^{*}=B\left(p^{*}\right)^{M}\left[1+C \ln \dot{\varepsilon}^{*}\right] \leq \sigma_{f}^{\max }
\end{aligned}
$$

where $A, B, C, M, N$ are material constants, $\sigma_{i}^{\max }$ and $\sigma_{f}^{\max }$ are the optional limits. The normalized pressure, $p^{*}$, and normalized maximum tensile hydrostatic pressure, $T^{*}$, are defined as $p^{*}=p / p_{H E L}$ and $T^{*}=T / P_{H E L}$, where $P, P_{H E L}, T$ are the actual pressure, pressure at the HEL and maximum tensile pressure that the material can withstand.

The damage initiation parameter, $\omega$, accumulates with plastic strain according to

$$
\omega=\sum \frac{\Delta \varepsilon^{p}}{\varepsilon_{f}^{p}(P)}
$$

where $\Delta \varepsilon^{p}$ is the increment in equivalent plastic strain and $\varepsilon_{f}^{p}(P)$ is the equivalent plastic strain to fracture under constant pressure, defined as $\varepsilon_{f}^{p}=D_{1}\left(P^{*}+T^{*}\right)^{D_{2}}$, where $D_{l}$ and $D_{2}$ are constants. JH-2 model assumes that the damage variable increases gradually with plastic deformation by setting $D=\omega$.

The equations for the pressure-density is described as

$$
\begin{array}{ll}
p(\zeta)=k_{1} \zeta+k_{2} \zeta^{2}+k_{3} \zeta^{3}+\Delta p ; & \text { compression } \\
p(\zeta)=k_{1} \zeta ; & \text { tension }
\end{array}
$$

where $\zeta=\rho / \rho_{0^{-}} 1$ and $\Delta p$ is an increment in the pressure and $k_{1}, k_{2}, k_{3}$ are material constants. The increment in pressure arises from the conversion of energy loss due to damage into internal energy.

\section{EXPERIMENTAL SETUP}

Polycrystalline TCO $\left(\mathrm{SnO}_{2}: \mathrm{F}\right)$ films were deposited on a $3.2 \mathrm{~mm}$-thick soda lime substrate using chemical vapor deposition (CVD) at $600^{\circ} \mathrm{C}$. Stack layers of polycrystalline $\mathrm{CdS}$ and $\mathrm{CdTe}$ were then sequentially thermally evaporated at $350^{\circ} \mathrm{C}$ substrate temperature. Film thicknesses of TCO, $\mathrm{CdS}$ and CdTe layers were found to be about $400 \mathrm{~nm}$, $200 \mathrm{~nm}$ and $2 \mu \mathrm{m}$ through ellipsometry measurements.

Experiments were carried out using a Nd:YAG laser. The system delivered 9-ns pulses at a $100 \mathrm{~Hz}$ repetition rate and a wavelength of $532 \mathrm{~nm}$. The samples were cleaned with acetone in an ultrasonic cleaner for 5 minutes and then rinsed with methanol and distilled water prior to processing. The sample was placed inside a metallic container sealed with quartz at both top and bottom to avoid hazardous plume during laser processing and collect the removed materials. This container was mounted on a three-axis 
translation stage and samples were irradiated by laser focused by a $20 \mathrm{X}$ objective lens. The focal plane was placed at the interface between TCO and CdS to create a circular beam spot with a diameter of around $50 \mu \mathrm{m}$. The samples were irradiated from the glass side by single pulse with various fluences from 0.4 to $6 \mathrm{~J} / \mathrm{cm}^{2}$ at a stationary spot, and then scribed with the fluence values from 1 to 3 $\mathrm{J} / \mathrm{cm}^{2}$ at different speeds (2 to $3.6 \mathrm{~mm} / \mathrm{s}$ ).

The treated samples were observed through SEM. Surface roughness and scribe profiles were measured by optical profilometry. The chemical components and cleanliness of laser processed samples were investigated by EDX. The generation of defects, such as material microstructure, micro cracks/voids and thermal effect after irradiation were analyzed by TEM.

\section{RESULTS AND DISCUSSION}

Figure 1 shows the schematic of $\mathrm{P} 2$ laser scribing on CdTe-based solar cells from the glass side under microexplosion mechanism. The removal mechanism can be divided into three steps: first, the laser has been absorbed by the entire and partial layers of $\mathrm{CdS}$ and $\mathrm{CdTe}$, respectively. The absorption depths of CdS (film thickness of 200nm) and CdTe (film thickness of $2 \mu \mathrm{m}$ ) at the wavelength of $532 \mathrm{~nm}$ are $\sim 1 \mu \mathrm{m}$ and $167 \mathrm{~nm}$, respectively. Second, as the pulse continues heating, the material in the absorption volume has been melted, vaporized and finally formed a plasma. Due to the confinement of substrate and solid CdTe, an intensive pressure induced by the plasma expansion will pull the solid CdTe material upwards and to delaminate from $\mathrm{CdS}$ layer. This is because the adhesion between $\mathrm{CdS}$ and CdTe layers is smaller than that between $\mathrm{SnO}_{2}: \mathrm{F}$ and $\mathrm{CdS}$ layers. Third, the deformed solid CdTe layer has been removed due to brittle fracture and the ablated material is also ejected with the pressure release to the ambient. Some resolidified molten CdS and undamaged $\mathrm{CdS}$ materials remain after the removal process. Due to the weak adhesion between CdS and CdTe, there is no CdTe has been melted at the boundary. In contrast, CdS removal is dominant by ablation. Therefore, thermal effect only occurs at this layer.

\section{Film removal under high fluences}

Figure 2 (a) shows the SEM image of film removal by single pulse irradiated at a fluence of $3 \mathrm{~J} / \mathrm{cm}^{2}$. It can be seen that a near-circular area with a diameter of around $80 \mu \mathrm{m}$ is scribed. A macro-crack occurs along the circumference of the scribed area. Magnified SEM image Fig. 2 (b) shows clearly that the macro-crack occurs at the boundary is partially broken, which may be due to the non-uniform beam energy distribution of the laser spot. The material at a high energy distributed area has been removed faster than the rest area, and then the trapped pressure between the film and substrate is released quickly. Therefore, the

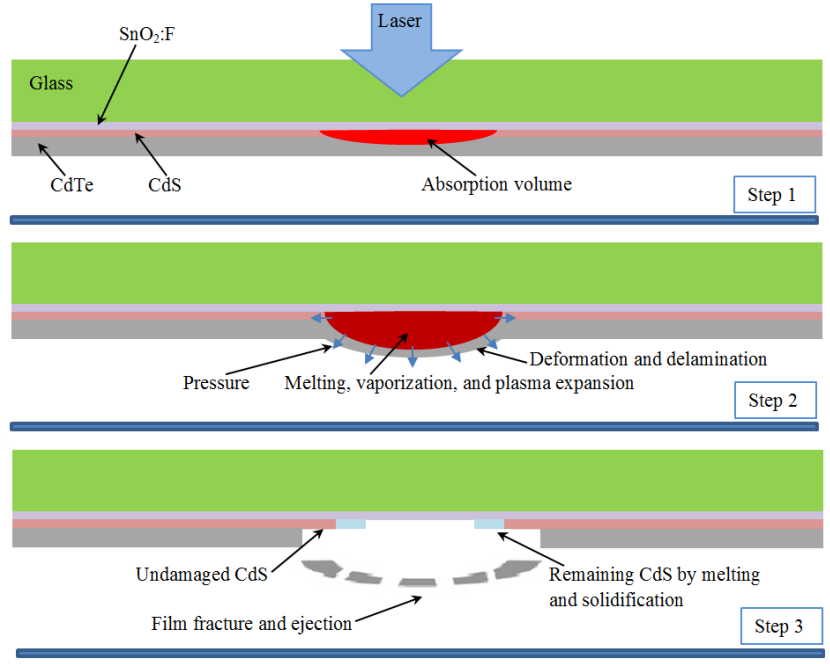

Figure 1. Schematic of film removal of $P 2$ laser scribing on CdTe-based solar cells under micro-explosion mechanism

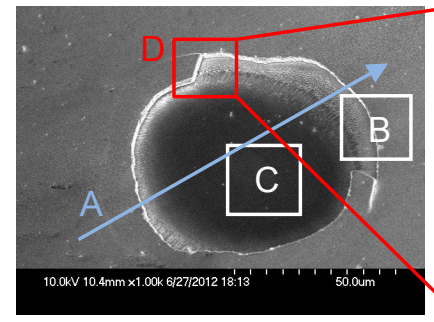

(a)

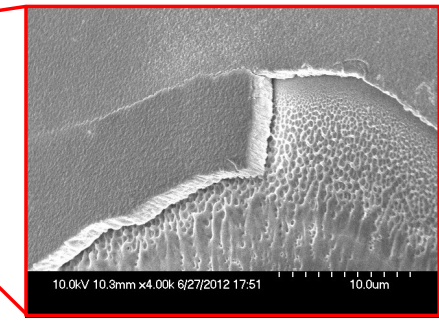

(b)

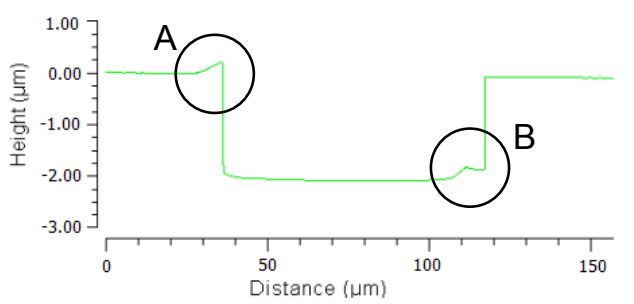

(c)

Figure 2. (a) SEM image of film removal by a pulse irradiated at a fluence of $3 \mathrm{~J} / \mathrm{cm}^{2}$; (b) magnified SEM image at square D; (c) Optical profilometry measurement along A

significantly reduced pressure is not strong enough to facture the rest CdTe material, instead, the pressure makes CdTe delaminate from the CdS layer and form as a macrocrack after processing. It is also observed that some material remains, which may include the undamaged $\mathrm{CdS}$ material and solidification of a liquid flow driven to the boundary by pressure releasing. Since the molten CdS layer is much thinner than the thickness of removal films, the pressure distribution at the boundary is almost parallel to the film surface, thus, the molten CdS material can only be push to the boundary. This is why from the optical profilometry measurement along A shown in Fig. 2(c), the circle B area shows a gradual increase to the boundary and 
the highest point occurs at the interface of solid/liquid CdS. Circle A shows the appearance of the macro-crack of CdTe film, and the reason why no delamination can be seen is because the detecting optical beam is perpendicular to the samples, so that the gap at the CdS/CdTe interface cannot be measured. The depth of film removal is measured to be $2 \mu \mathrm{m}$ at the scribed center with a diameter of around $60 \mu \mathrm{m}$, and vertical sidewalls are formed after processing. EDX line profile along A is shown in Fig. 3. It can be seen that no other elements except $\mathrm{Si}$ and $\mathrm{Sn}$ in region $\mathrm{B}$, which indicates that $\mathrm{CdS}$ and CdTe are completed removed in this region and no inter-diffusion of S into the TCO layer. The co-existing of $\mathrm{Si}$ and $\mathrm{Sn}$ is due to the larger electron penetration depths of $\mathrm{SnO}_{2}: \mathrm{F}$ compared to its thickness[13]. In region $\mathrm{A}(\sim 10 \mu \mathrm{m})-$ macro-crack broken area, elements of $\mathrm{Cd}$ and $\mathrm{S}$ are detected besides $\mathrm{Sn}$ and $\mathrm{Si}$. This demonstrates that the only $\mathrm{CdS}$ remains, which matches the mechanism described in Fig. 1 that higher adhesion occurs at the interface between $\mathrm{CdS}$ and TCO layers.

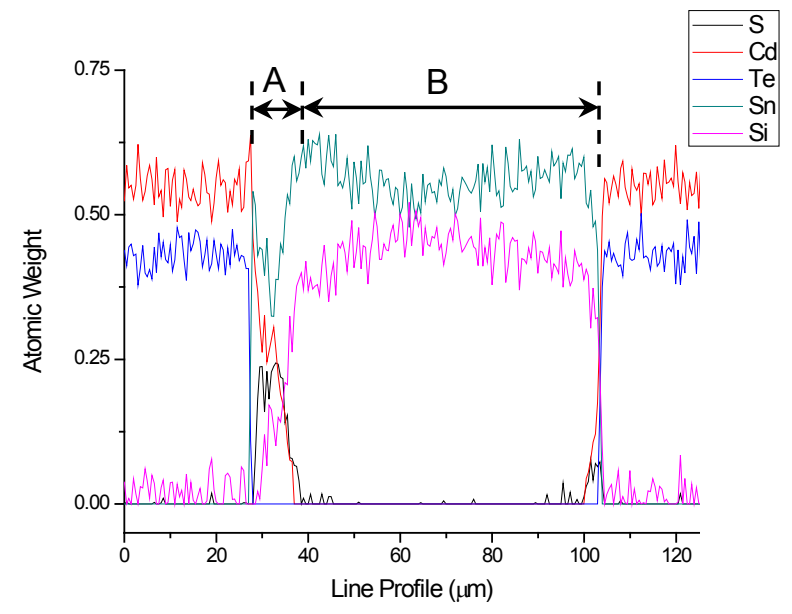

Figure 3. EDX line profile scanning along A in Fig. 2 (a), showing a clean removal at the center, and small amount of remaining material is CdS and no inter-diffusion occurs at the interface of $\mathrm{TCO} / \mathrm{CdS}$

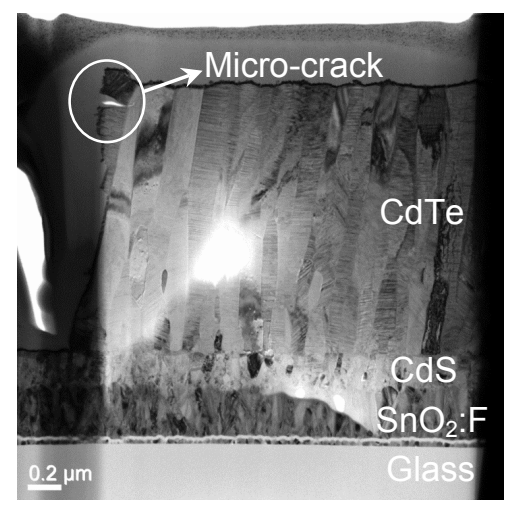

Figure 4. Cross-sectional TEM image of scribe boundary at square B shown in Fig.2 (a)

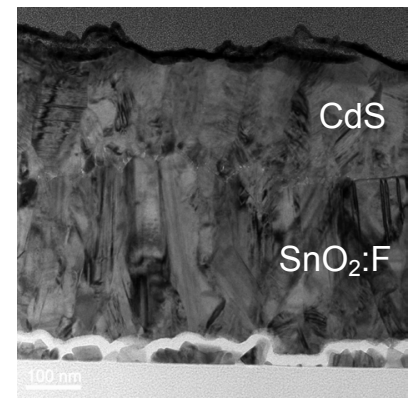

(a) scribe boundary

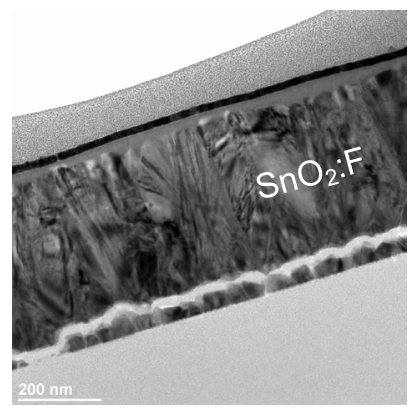

(b) scribe center
Figure 5. Cross-sectional TEM images of (a) near scribe boundary and (b) scribe center region $\mathrm{C}$ in Fig. 2 (a)

Figure 4 depicts the cross-sectional TEM image at the scribe boundary B shown in Fig. 2 (a). CdTe grains grow epitaxially on CdS grains with a dominant orientation of (111) and the columnar CdTe grains with microtwins results in a poor photocurrent and high series resistance [2]. Vere et al. [25] showed twinning is an intrinsic feature of the zincblende-structure materials with low stacking fault energy, such as II-V (CdTe) and III-V semiconductors. It originates on the liquid/solid interface during growth, where group of atoms deposited at a point or points on the interface are misorientated with respect to the reminder. It can be seen that CdTe is removed mechanically instead of thermal ablation. The pressure induced by plasma expansion causes the deformation of CdTe layer and delamination from the $\mathrm{CdS}$ layer. Brittle fracture sequentially occurs at high stress concentration points at the constraint boundary. Cracks initialize at the bottom of the CdTe layer and propagate to the top surface, since the deformed geometry of boundaries causes higher material strains at the bottom compared to the top surface. The crack propagates along the grain boundary (intergranular fracture) at the bottom, because impurities usually segregate at the $\mathrm{CdS} / \mathrm{CdTe}$ interface in the beginning of deposition, which weaken and embrittle the grain boundaries. When crack propagates to the top surface, it passes through grains (transgranular fracture) by cutting across twins. This may be caused by the direction change of the applied stress due to the film deformation and grain geometries. The atomic bonding energy becomes smaller for the atoms near the top surface, and a lateral nano-crack occurs at this point simultaneously. Figure 5 (a) shows that the remaining $\mathrm{CdS}$ layer near the sidewall has the same microstructure and thickness compared to the undamaged $\mathrm{CdS}$ in Fig. 4. Likewise, the TEM image of Fig. 5 (b) taken at the scribe center (region $\mathrm{C}$ in Fig. 2 (a)) exhibits that only $\mathrm{SnO}_{2}: \mathrm{F}$ layer remains and no $\mathrm{SnO}_{2}: \mathrm{F}$ material has been damaged after scribing. Therefore, it can be concluded that $\mathrm{CdS}$ material has been ablated in the laser effected area. In the meanwhile, CdTe delaminates from CdS layer and is removed due to the brittle fracture caused by the plasmainduced pressure. The thermal effect only occurs at the 
layer of CdS and no damage of $\mathrm{SnO}_{2}: \mathrm{F}$ or inter-diffusion of sulfur is observed after processing.

\section{Film removal under low fluences}

Figure 6 shows the SEM image of film removal irradiated by one pulse at a fluence of $1 \mathrm{~J} / \mathrm{cm}^{2}$. It can be seen that a circular scribed area with a diameter of around $60 \mu \mathrm{m}$ with much more remaining material compared to that scribed under $3 \mathrm{~J} / \mathrm{cm}^{2}$. In addition, no macro-crack is observed in this condition. In order to understand the removal mechanism, cross-sectional TEM images at the scribe boundary B' are carried out as shown in Fig. 7 (a). It can be seen that a micro-crack and some nano-voids appear at the $\mathrm{CdS} / \mathrm{CdTe}$ interface. The CdTe layer under a lower fluence needs more time to be completely fractured. Thus, when a crack initializes at the bottom and starts propagating to the top surface, there is enough time for the pressure to causes a lateral crack and several nano-voids along the interface of $\mathrm{CdS}$ and CdTe due to the weak adhesion before the entire layer has been removed. Similarly to the film removal at $3 \mathrm{~J} / \mathrm{cm}^{2}$, the remaining CdS is not thermal affected at the removal boundary and vertical sidewalls are formed. Figure 7 (b) shows the magnified TEM image near crack tip. It can be observed that the high stress concentration at the crack tip cause change of atomic structures in both $\mathrm{CdS}$ and $\mathrm{CdTe}$ crystals. Moreover, nano-cracks are founded around the nano-voids (not shown). These phenomena suggest that those dislocation slips and deformation twinning, caused by the atomic structure rearrangements, may play an important role in the final stages to the material failure [26] and the further reduction of electric output. Cross-sectional TEM image taken at region $\mathrm{C}^{\prime}$ in Fig. 6 is shown in Fig. 8 (a). It can be seen that both microstructures and thickness of $\mathrm{CdS}$ layer has been changed due to the thermal effect. The arrow shows the direction to the removal boundary. The molten $\mathrm{CdS}$ material solidifies and the grain grows vertically from $\mathrm{SnO}_{2}: \mathrm{F}$ layers near the boundary, however, the grains close to the center shown in Fig. 8 (b) exhibit different microstructures and nano-bubbles near the interface. The grain size in this region is much smaller (a few nanometers) based on high-resolution TEM images (not shown). The thermal gradient in the molten pool drives the motion of liquid CdS provoking the deformation of the surface, and the pressure differences created at a curved interface support the evolution of the deformation on the liquid surface known as capillary waves [17]. However, due to the plasma expansion and micro-explosion process, the pressure propagated along horizontal direction pushes more liquid CdS to the boundary and forms the different amplitudes along the capillary wave. The liquid CdS close to the center under stronger pressure travels a longer distance, thus, there is no enough time for a similar recrystallization process to that happened near the boundary. Moreover, decomposition of CdS occurs due to the high temperature, and sulfur can be quickly oxidized

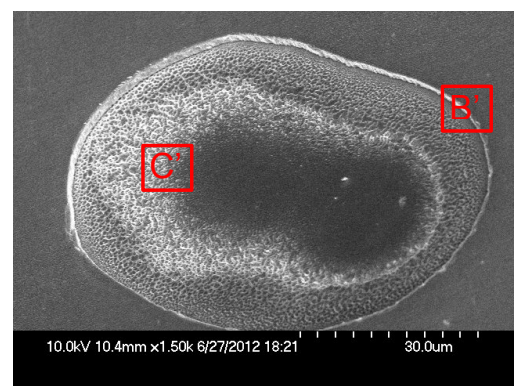

Figure 6. SEM image of film removal by a pulse irradiated at a fluence of $1 \mathrm{~J} / \mathrm{cm}^{2}$, showing much more CdS remaining after processing

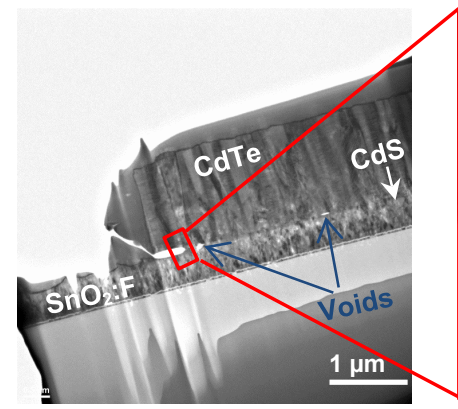

(a)

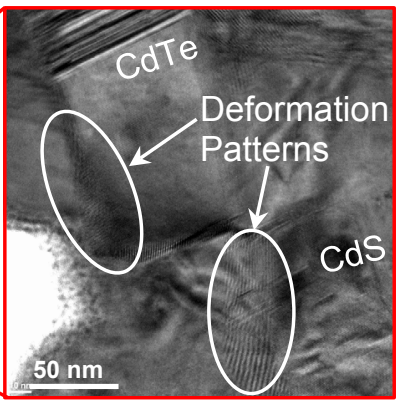

(b)
Figure 7. Cross-sectional TEM images of (a) scribe boundary at square B' shown in Fig.6 and (b) magnified image at the crack tip (a)

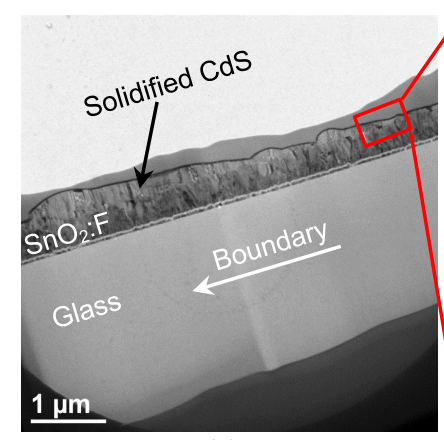

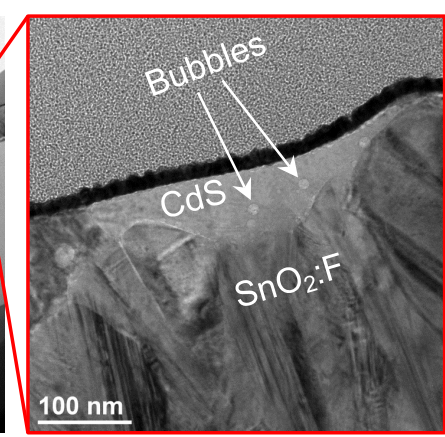

(b)
Figure 8. (a) TEM images taken at region $C^{\prime}$ in Fig. 6 and (b) magnified TEM image at the interface between $\mathrm{CdS}$ and $\mathrm{SnO}_{2}: \mathrm{F}$ layers.

and formed as gaseous phase. Sulfur oxide either evaporates to the environment or stay inside the CdS matrix to form as bubbles after solidification.

Overall, scribing under both high and low fluences can achieve a complete film removal and no damage of $\mathrm{SnO}_{2}: \mathrm{F}$ or interdiffusion of sulfur occurs after processing. However, some CdS remains and partial of the material has been molted and recrystallized. This will affect the next-step contact layer deposition in the solar cell fabrication process. In addition, the ultra-fast heating process could introduce 
supercooling during recrystallization, which causes defect generation and reduces the crystal quality. Moreover, the bubbles formed by sulfur oxide will also introduce abnormal heating and eventually damage the cells [19]. Scribing under a high fluence can cause macro-cracks due to the pulse imperfection and micro-cracks near the top surface which may decrease the active area of absorber layers. However, scribing under a low fluence can lead to more remaining CdS material, micro-cracks and nano-voids at the $\mathrm{CdS} / \mathrm{CdTe}$ interface, and dislocation slips and deformation twinning at the crack tips. This makes the scribe quality even worse compared to that irradiated at a higher fluence, since more defects are introduced.

\section{Parametric study and line scribing process}

In order to optimize the scribing conditions, a parametric study is carried out for the dependence of scribe area and remaining $\mathrm{CdS}$ on the irradiation fluences $\left(0.8 \mathrm{~J} / \mathrm{cm}^{2}\right.$ to $\left.6 \mathrm{~J} / \mathrm{cm}^{2}\right)$ as shown in Fig. 9. Since the pulse shape is irregular, the measurement of scribe areas is taken rather than in diameter. From the observation under SEM and optical profilometry, the threshold for complete removal is $0.8 \mathrm{~J} / \mathrm{cm}^{2}$, and the ratio between remaining material and total removal area becomes much less and macro-crack occurs after $1.6 \mathrm{~J} / \mathrm{cm}^{2}$, and $\mathrm{SnO}_{2}: \mathrm{F}$ layer has been damaged at $6 \mathrm{~J} / \mathrm{cm}^{2}$. Therefore, the scribing condition can be focused on the fluences from $0.8 \mathrm{~J} / \mathrm{cm}^{2}$ to $6 \mathrm{~J} / \mathrm{cm}^{2}$, where low fluence range represents $0.8 \mathrm{~J} / \mathrm{cm}^{2}$ to $1.6 \mathrm{~J} / \mathrm{cm}^{2}$ and high fluence range represents $1.6 \mathrm{~J} / \mathrm{cm}^{2}$ to $6 \mathrm{~J} / \mathrm{cm}^{2}$. It can be seen that the scribe areas are close to $4100 \mu \mathrm{m}^{2}$ in the low fluence range, and $4300 \mu \mathrm{m}^{2}$ from $1.6 \mathrm{~J} / \mathrm{cm}^{2}$ to $3.5 \mathrm{~J} / \mathrm{cm}^{2}$. This difference is because in the low fluence range, partial of the CdTe film delaminated from CdS layer cannot be completely removed as shown in Fig. 7. In contrast, the boundary is clear under the high fluence range as shown in Fig. 4. The trend of scribe area shows a linear increase with increasing of fluences from $3.5 \mathrm{~J} / \mathrm{cm}^{2}$ to $6 \mathrm{~J} / \mathrm{cm}^{2}$. This is due to absorption volumes become larger under higher fluences, therefore, the sequential plasma volume increase and more material will be removed. In addition, the increased pressure could also cause $\mathrm{CdS}$ material fracture, which leads to the increase of scribe areas. The trend of the percentage of remaining CdS area is linear decrease with increasing of fluences in the low fluence range and keeps a constant in the high fluence range. The laser-induced pressure becomes larger and larger with increasing fluences during micro-explosion processes, thus, more liquid $\mathrm{CdS}$ is driven to the boundary and less $\mathrm{CdTe}$ delaminates from the $\mathrm{CdS}$ layer due to faster brittle-fracture responses. Until the pressure reaches a threshold, all liquid $\mathrm{CdS}$ moves to the boundary and cannot flow out of the removal cavity by the prevention of CdTe layer.

In order to optimize the line scribing condition, samples are processed at the fluence values from 1 to 3 $\mathrm{J} / \mathrm{cm}^{2}$ with different speeds from 1 to $4 \mathrm{~mm} / \mathrm{s}$, which equivalent to the pulse overlapping from $80 \%$ to $20 \%$. Among the complete removal conditions, the highest speed occurs at $2 \mathrm{~mm} / \mathrm{s}$ under a fluence of $3 \mathrm{~J} / \mathrm{cm}^{2}$ as shown in Fig. 10 (a). It can be seen a clean line is formed in a width of $70 \mu \mathrm{m}$. Fig. 10 (b) shows that macro-cracks are removed by the next overlapped pulse at the removal boundary. Although the scribing speed is not in the desirable range $(\mathrm{m} / \mathrm{s})$ due to the limitation of pulse repetition rate, the experimental results can still be referenced with different laser facilities. Therefore, it can be concluded that the condition of $3 \mathrm{~J} / \mathrm{cm}^{2}$ with a pulse overlapping of $\sim 60 \%$ is the optimal processing condition for P2 laser scribing of CdTe-based solar cells.

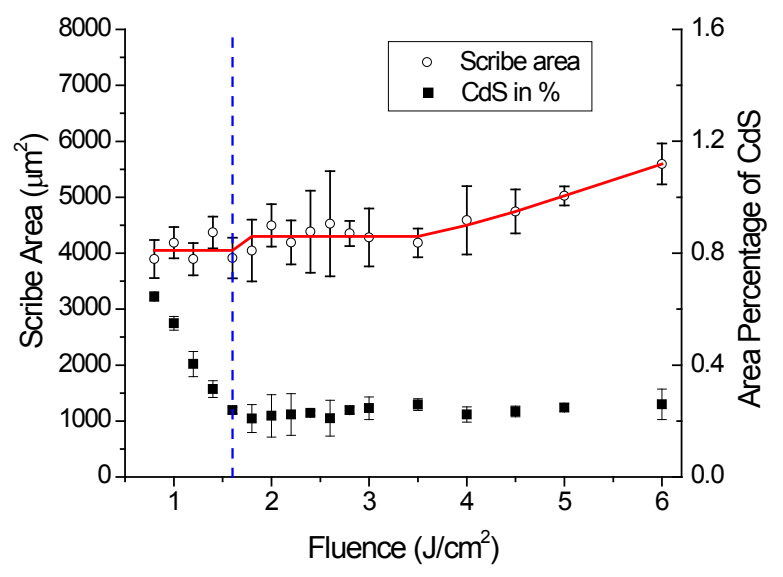

Figure 9. Dependence of scribe area and remaining $\mathrm{CdS}$ on laser irradiation conditions

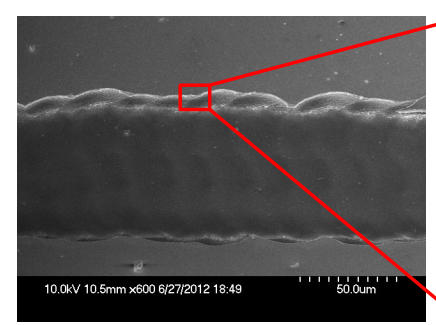

(a)

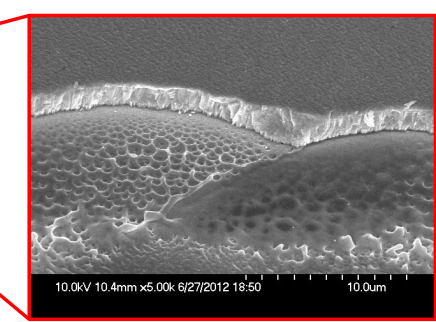

(b)
Figure 10. SEM images of (a) line scribing at a fluence of $3 \mathrm{~J} / \mathrm{cm}^{2}$ and a speed of $2 \mathrm{~mm} / \mathrm{s}$, (b) magnified image at scribe boundary, showing macro-cracks are removed due to the pulse overlapping

\section{SIMULATION ON FILM REMOVAL}

Two-dimensional thermal and mechanical models are setup based on the previous study [13], except adding a CdS layer in the thermal model to determine the plasma volume and considering dynamic response in CdTe fracture criteria discussed before. Figure 11 shows the temperature distribution of $\mathrm{CdTe} / \mathrm{CdS} / \mathrm{SnO}_{2}: \mathrm{F}$ stack layers on glass substrate as a result of the glass-side laser irradiation at a fluence of $1 \mathrm{Jcm}^{2}$. The thicknesses of different layers are 
$2 \mu \mathrm{m}, 200 \mathrm{~nm}, 400 \mathrm{~nm}$ and $50 \mu \mathrm{m}$, respectively. The laser pulse is $9 \mathrm{~ns}$ in duration, Gaussian-distributed, $50 \mu \mathrm{m}$ in spot size and $532 \mathrm{~nm}$ in wavelength. The thermal analysis is carried out with the consideration of energy loss due to the reflection at the interfaces of glass/air, glass $/ \mathrm{SnO}_{2}: \mathrm{F}$ and $\mathrm{SnO}_{2}: \mathrm{F} / \mathrm{CdS}$, heat convection at the boundary, as well as the absorption by the glass substrate and $\mathrm{SnO}_{2}: \mathrm{F}$ layer. Material properties can be found in ref. [13]. It can be observed that the absorbing volume is confined between the CdTe and $\mathrm{SnO}_{2}: \mathrm{F}$ layers, and the temperature is uniformly distributed in $\mathrm{CdS}$ layer due to the different absorption depths discussed before. This highly confined energy increases temperature up to $8000 \mathrm{~K}$ at this condition, which is higher than the vaporization temperatures of both $\mathrm{CdTe}$ and $\mathrm{CdS}$, therefore, plasma has been generated. The temporal and spatial distributions of plasma-expansion-induced pressure are then calculated based on ref. [13] and the constant fraction $\alpha$ is kept 0.04 to match the removal threshold compared to experimental results.

Film removal is presented in a mechanical model with the pressure incorporated on CdTe layer at the interface. A $10 \mathrm{~nm}$-thick layer of cohesive elements is implemented to consider the traction stresses at the interface. The cohesive layer is governed by the traction separation law described in [13], and serves the purpose of simulating the process in which the CdTe film lifts up and delaminates from the CdS layer caused by the plasma expansion. In order to simplify the problem, CdS is neglected from the mechanical model. Because CdS layer is dominantly removed by ablation based on experimental results, there is no effect of mechanical fracture on this layer during micro-explosion process. Since adhesion becomes zero at the plasma area, an open cavity at the center of the cohesive layer, representing plasma width, is implemented. The values of plasma width are extracted from the thermal model. The pressure is applied at the interface of CdTe and cohesive layer and the boundaries are fixed.

Figure 12 shows the complete film removal with a fluence of $1 \mathrm{~J} / \mathrm{cm}^{2}$ at $71.6 \mathrm{~ns}$. It can be seen that a scribe area with a radius of $28.7 \mu \mathrm{m}$ and a further micro-crack of 4.2 $\mu \mathrm{m}$ at the interface. Therefore, film delamination occurs compared to the initial plasma width of $41.6 \mu \mathrm{m}$. This matches the experimental result as shown in Fig. 7 (a). The simulation result shows that the strain rate of failed elements is up to $10^{5} \mathrm{~s}^{-1}$. Therefore, the material dynamic response is necessary to be implemented. The film is removed mainly due to the tensile stress, and the ratedependent response has much less influence on the tensile yield stress than the compressive yield stress for brittle materials. However, the implemented criteria can still affect the compressive failure and lead to more accurate element deformations compared the static fracture laws. The film deformation causes tensile stress to the elements at the top center and bottom boundary. Therefore, the fracture occurs preferentially at these points. Since the pressure is not strong enough to break the entire layer immediately, a

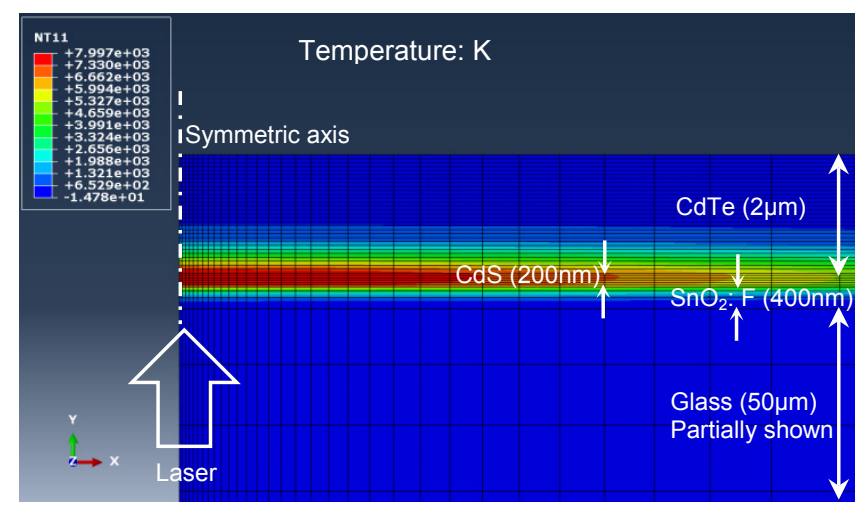

Figure 11. Simulation result of temperature distribution of $\mathrm{CdTe} / \mathrm{CdS} / \mathrm{SnO}_{2}: \mathrm{F} /$ glass multilayer thermal model at a fluence of $1 \mathrm{~J} / \mathrm{cm}^{2}$

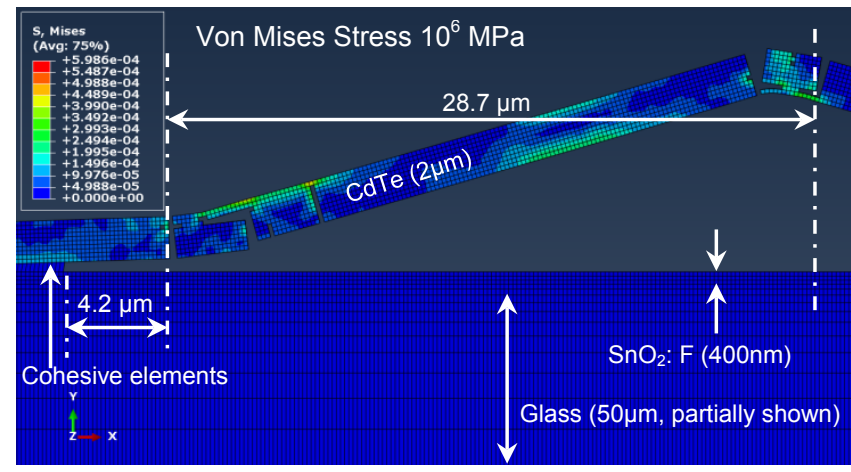

Figure 12. Complete film removal with a fluence of $1 \mathrm{~J} / \mathrm{cm}^{2}$ at $71.6 \mathrm{~ns}$, the scribe radius is $28.7 \mu \mathrm{m}$ and micro-crack propagates $4.2 \mu \mathrm{m}$ through the interface. Deformation scale is $1.5 \mathrm{X}$ for viewing clarity

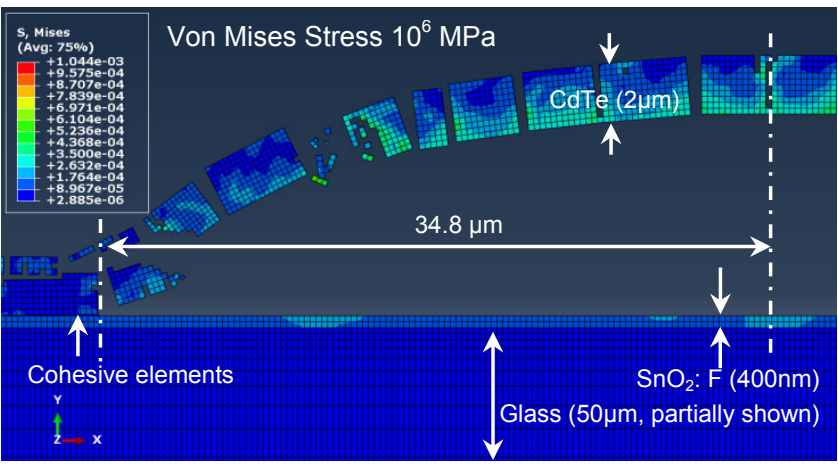

Figure 13. Complete film removal with a fluence of $3 \mathrm{~J} / \mathrm{cm}^{2}$ at $30 \mathrm{~ns}$, the scribe radius is $34.8 \mu \mathrm{m}$ and micro-crack occurs near the top surface. Deformation scale is $0.3 \mathrm{X}$ for viewing clarity

further micro-crack along the interface occurs due to its weak adhesion. The film still remains large pieces when the removal is completed and the breaks occur at both center and boundary. Fig. 13 shows the film removal at a fluence 
of $3 \mathrm{~J} / \mathrm{cm}^{2}$. It can be seen that a scribe area with a radius of $34.8 \mu \mathrm{m}$ (plasma width is $58.8 \mu \mathrm{m}$ ) and this removal only takes 30ns, which is less than one half of that happened at $1 \mathrm{~J} / \mathrm{cm}^{2}$. Similarly, initial fractures occur at the bottom boundary and top center. However, since the pressure is strong enough to cause further deformation, some elements between the boundary and center also achieve their fracture limit. Likewise, more and more elements between those "pre-fracture" points fail and finally, the film becomes many small pieces after removal. At the boundary, microcracks occur near the top surface rather than at the interface. During the crack propagates through the boundary from bottom to the top, the stress induced by the pressure and film deformation is strong enough to fracture their neighboring elements so that the micro-crack preferentially occurs at a relatively weak bonding area near the top surface, which has been presented in Fig. 4. Fig. 14 shows the optical microscopy image of collected removal material after laser scribing. It can be observed that the film is broken into many pieces in a range of a few micrometers to around $60 \mu \mathrm{m}$ in length. This shows a good agreement with the simulation results discussed above.

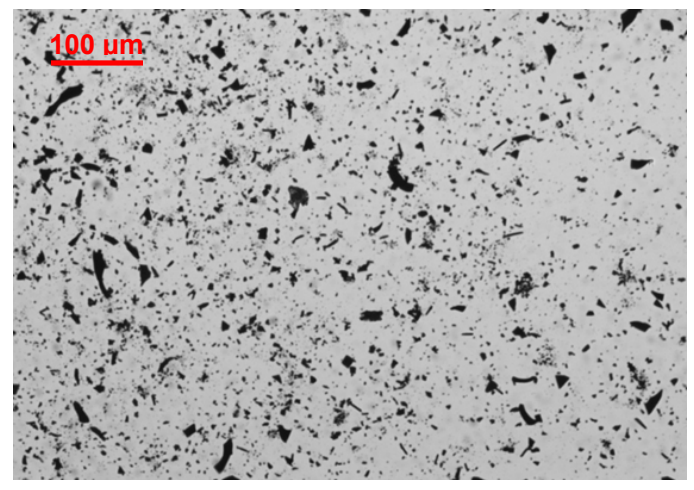

Figure 14. Optical microscopy image of collected removed materials, showing small and large pieces of removal material which are produced by high and low fluences.

Figure 15 shows the simulation results of dependence of plasma width, scribe diameter and remaining $\mathrm{CdS}$ on laser irradiation fluences. It can be seen that the plasma width increases to around $55 \mu \mathrm{m}$ at $2.25 \mathrm{~J} / \mathrm{cm}^{2}$ and maintains almost a constant afterwards. Compared to the spot size $(50 \mu \mathrm{m})$, only $2.5 \mu \mathrm{m}$-wide $\mathrm{CdS}$ material in lateral direction has reached the vaporization temperature beyond the laser irradiation area. This presents the laser-induced heat can only be transferred laterally close to CdTe film thickness $(2 \mu \mathrm{m})$, because it is dissipated quickly to the ambient at the top of CdTe layer. The scribe diameter shows a similar trend to that of plasma width. The simulation result shows the high fluence starts from $2.25 \mathrm{~J} / \mathrm{cm}^{2}$, where the microcrack at the interface (as shown in Fig. 12) disappears and film is fractured in many small pieces. In the high fluence range, the scribe width keeps constant, because the pressure is strong enough to fracture the entire film immediately and there is no time to lead to further deformation and delamination. In contrast, in the low fluence range, more $\mathrm{CdTe}$ material delaminates from the layer underneath since it takes more time for the film to deform before entire layer is fractured. The percentage of $\mathrm{CdS}$ material shows a similar trend to the experimental result shown in Fig. 9, more CdS remains in the low fluence range and finally it keeps a constant in the high fluence range. The discrepancy of an underestimation in the simulation could be caused by ignoring the melting of CdS. However, the overall fluence dependence on film removal by micro-explosion is accurately captured by the simulation.

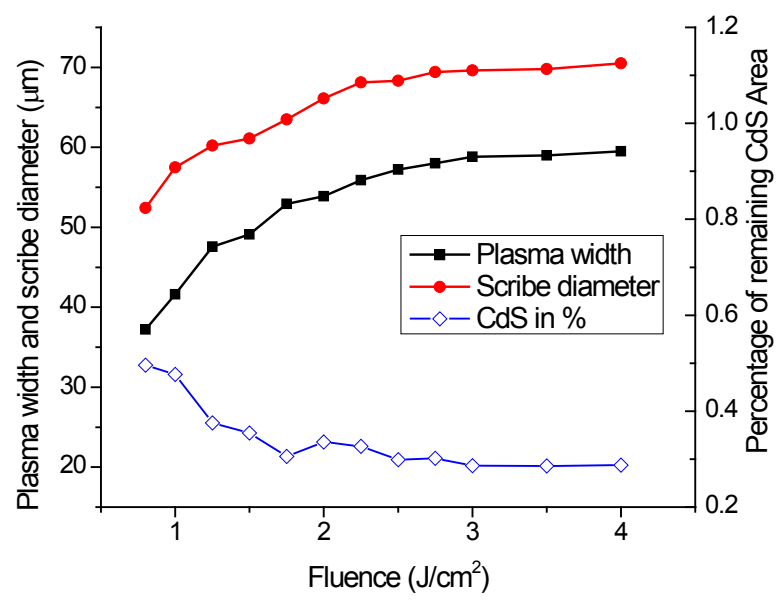

Figure 15. Simulation results of dependence of plasma width, scribe diameter, and remaining $\mathrm{CdS}$ on laser irradiation fluences.

\section{CONCLUSION}

In conclusion, it has been demonstrated that successfully selective film removal with well-defined sidewalls and little thermal effect is achieved by glass-side laser scribing of CdTe-based multilayer solar cells. The micro-explosion mechanism only leads melting and recrystallization on CdS layer, which could cause defect generation under the supercooling process. CdS material is removed dominantly through ablation because its absorption depth is much larger than the thickness. In contrast, the rest of laser energy is fully absorbed by a shallow layer in the CdTe film. Due to the confinement of solid CdTe and the substrate, a shock wave is generated caused by the plasma expansion and CdTe material is removed through brittle fracture by the induced pressure. CdTe film delimitation from the CdS layer is also observed due to the weaker adhesion between these two layers compared to other interfaces, which will affect the next-step contact layer deposition in the solar cell fabrication process. Furthermore, other defects are characterized under both low and high fluence ranges. These defects, including nano- 
bubbles caused by sulfur oxidation, micro-cracks, dislocation slip and deformation twinning formed at crack tips due to the atomic structure rearrangement, could further induce abnormal heating and poor photocurrent. The optimal condition (with minimum defects and remaining CdS material) of glass-side laser scribing is determined to be at a fluence of $3 \mathrm{~J} / \mathrm{cm}^{2}$ with a speed of $2 \mathrm{~mm} / \mathrm{s}$ based on parametric studies. The low scribing speed is limited by the pulse repetition rate, however, the experimental results are still valuable for the investigation on different required laser facilities. Finally, numerical finite element models are developed for P2 scribing based on micro-explosion mechanism. Good agreements with experimental results show that the simulation is capable of predicting the material removal dynamics and fracture behavior of CdTe.

\section{ACKNOWLEDGMENTS}

Research carried out in part at the Center for Functional Nanomaterials, Brookhaven National Laboratory, which is supported by the U.S. Department of Energy, Office of Basic Energy Sciences, under Contract No. DE-AC02-98CH10886. The use of material characterization equipment at Materials Research Science and Engineering Center, Columbia University is also gratefully acknowledged. Thanks are due to Drs. Marco Leona and Pablo Londero, Department of Scientific Research, the Metropolitan Museum of Art, for processing equipment use.

\section{REFERENCES}

[1] Dhere, R.G., et al., 2011. CdTe solar cell with industrial $\mathrm{Al}: \mathrm{ZnO}$ on soda-lime glass. Thin Solid Films, 519/21: $7142-7145$.

[2] Luque, A., Hegedus, S., 2003. Handbook of Photovoltaic Science and Engineering. Wiley, UK.

[3] Booth, H., 2010. Laser processing in industrial solar module manufacturing. Journal of Laser Micro/ Nanoengineering, 5: 183-191.

[4] Compaan, A., Matulionis, S., 2000. Laser scribing of polycrystalline thin films. Opt. Lasers Eng., 34: 15-45.

[5] Murison, R., et al., 2010. CIGS P1, P2 and P3 laser scribing with an innovative fiber laser. $35^{\text {th }}$ IEEE Photovoltaic Specialists Conf., 179-184.

[6] Bovatsek, J., et al, 2010. Thin film removal mechanisms in ns-laser processing of photovoltaic materials. Thin Solid Films, 518: 2897-2904.

[7] Dunsky, C., Colville, F., 2008. Scribing thin-film solar panels. Industrial Laser Solutions for Manufacturing.

[8] Gecys, P, Raciukaitis, G., 2010. Scribing of a-Si thin film solar cells with picoseconds laser. Eur. Phys. J. Appl. Phys., 51: 33209.

[9] Wang, W., et al., 2010. Comparison of femtosecond laser-induced front- and rear-side ablation of films. Proc. Inst. Mech. Eng., Part B: J. Eng. Manuf., 225: 520-527.
[10] Beyer, S., Tonrnari, V., Gornicki, D., 2003. Comparison of laser induced front- and rear side ablation. Proc. SPIE, 5063: 202-207.

[11] Sano, T., Yamada, H., Nakayama, T., Miyamoto, I., 2002. Laser induced rear ablation of metal thin films. Proc. SPIE, 4426: 70-73.

[12] Matylitsky, V.V., Huber, H., Kopf, D., 2011. Selective removal of transparent conductive oxide layers with ultrashort laser pulses: front- vs. back-side ablation. Int. Congr. App. Lasers Electro-Opt., M903: 1022-1027.

[13] Wang, H., Hsu, S., Tan, H., Yao, Y.L., Chen, H., Azer, M., 2012. Predictive modeling for glass-side laser scribing of thin film photovoltaic cells. 40th North American Manuf. Research Conf., Notre Dame, In.

[14] Shinohara, W., et al., 2006. Applications of laser patterning to fabricate innovative thin-film silicon solar cells. Proceedings of SPIE, 6107.

[15] Kontgers, M., et al., 2010. Quantifying the risk of power loss in PV modules due to micro cracks. 25th European Photovoltaic Solar Energy Conference, Spain.

[16] Tull, B.R., et al., 2006. Silicon surface morphologies after femtosecond laser irradiation. MRS Bulletin, 31/8:626-633.

[17] Wang, H., Lusquiños, F., Yao, Y.L., 2012. Effect of hydrogen on surface texturing and crystallization of a$\mathrm{Si}: \mathrm{H}$ thin film irradiated by excimer laser, Applied Physics A, 107/2: 307-320.

[18] Wang, H., Kongsuwan, P., Satoh, G., Yao, Y.L., 2012. Femtosecond laser-induced simultaneous surface texturing and crystallization of a-Si:H thin film: absorption and crystallinity. ASME Trans., Journal of Manu. Science and Engineering, 134/3: 031006.

[19] Acciania, G., Falcone, O., Vergura, S., 2010. Defects in poly-silicon and amorphous silicon solar cells. Intl. Conf. on Renewable Energy and Power Quality, Spain.

[20] Golovan, L.A., Kashkarov, P.K., Timoshenko, V.Y., 1996. Laser-induced melting and defect formation in cadmium telluride. Laser Physics, 6/5: 925-927.

[21] Emel'yanov, V.I., Kashkarov, P.K., 1992. Laserinduced defect formation in semiconductors. Applied Physics A, 55: 161-166.

[22] Addession, F.L., Johnson, J.N., 1990. A constitutive model for the dynamic response of brittle materials. Journal of Applied Physics, 67/7: 3275-3286.

[23] Holmquist, T.J., Johnson, G.R., 1994. An improved computational constitutive model for brittle materials. AIP Conf. Proc. 309: 981-984.

[24] Holmquist, T.J., Johnson, G.R., 2005. Characterization and evaluation of silicon carbide for high-velocity impact. Journal of Applied Physics, 97: 093502.

[25] Vere, A.W., Cole, S., Williams, D.J., 1983. The origins of twinning in CdTe. J. of Elec. Mat, 12/3: 551-561.

[26] Hai, S., Tadmor, E.B., 2003. Deformation twinning at aluminum crack tips. Acta Materialia, 51: 117-131. 